\title{
Organometallics Communication
}

\author{
The First Mixed Valent Antimony(III/V) Oxo Clusters \\ $\left(2,6-\mathrm{Mes}_{2} \mathrm{C}_{6} \mathrm{H}_{3} \mathrm{Sb}\right)_{2}(\mathrm{ClSb})_{4} \mathrm{O}_{8}$ and $\left(2,6-\mathrm{Mes}_{2} \mathrm{C}_{6} \mathrm{H}_{3} \mathrm{Sb}\right)_{4}(\mathrm{ClSb})_{4}(\mathrm{HOSb})_{2} \mathrm{O}_{14}{ }^{\dagger}$ \\ Jens Beckmann, ${ }^{*} a$ Timm Heek $^{a}$ and Masashi Takahashi ${ }^{b}$ \\ a Institut für Chemie und Biochemie, Freie Universität Berlin, Germany \\ ${ }^{\mathrm{b}}$ Department of Chemistry, Faculty of Science, Toho University, Miyama, Funabashi, \\ Chiba 274, Japan.
}

\section{Supporting Information:}

Figure S1. Molecular structure of 2,6- $\mathrm{Mes}_{2} \mathrm{C}_{6} \mathrm{H}_{3} \mathrm{SbCl}_{2}(\mathbf{1}) \cdot 0.5 \mathrm{C}_{4} \mathrm{H}_{10} \mathrm{O}$.

Figure S2. Molecular structure of 2,6- $\mathrm{Mes}_{2} \mathrm{C}_{6} \mathrm{H}_{3} \mathrm{SbCl}_{2}(\mathbf{1}) \cdot \mathrm{SbCl}_{3}$.

Figure S3. Inorganic core of $\left(2,6-\mathrm{Mes}_{2} \mathrm{C}_{6} \mathrm{H}_{3} \mathrm{Sb}^{\mathrm{V}}\right)_{2}\left(\mathrm{ClSb}^{\mathrm{III}}\right)_{4} \mathrm{O}_{8}(3)$ illustrating the two major disordered fragments.

Figure S4. $\quad{ }^{121} \mathrm{Sb}$ Mößbauer spectrum of $\left(2,6-\mathrm{Mes}_{2} \mathrm{C}_{6} \mathrm{H}_{3} \mathrm{Sb}^{\mathbf{V}}\right)_{2}\left(\mathrm{ClSb}^{\text {III }}\right)_{4} \mathrm{O}_{8}$.

\footnotetext{
$\dagger$ This work contains part of the Diploma thesis of Timm Heek

* To whom correspondence should be addressed. E-mail: beckmann@ chemie.fu-berlin.de. Fax: ++49-3083852440
} 


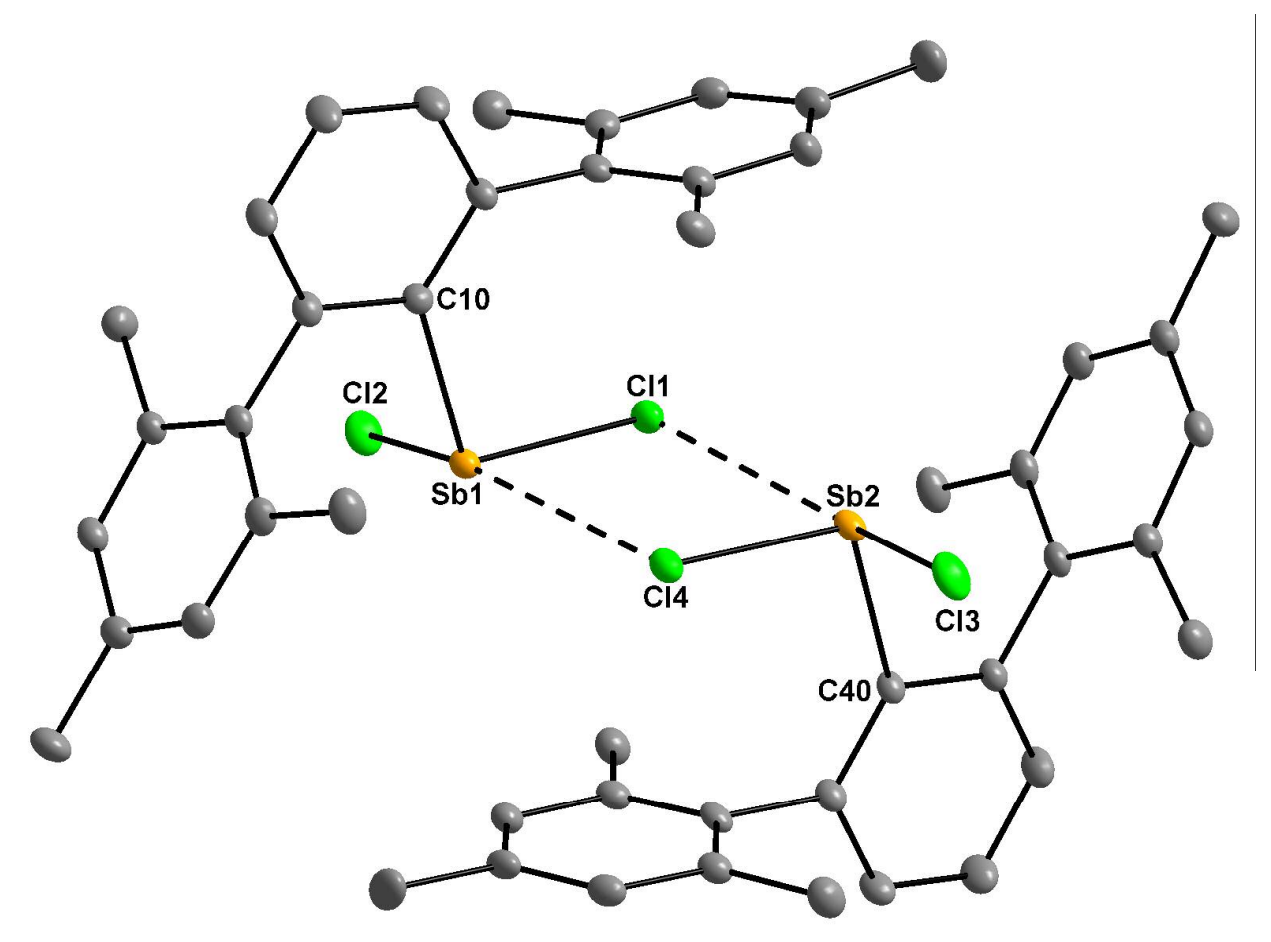

Figure S1. Molecular structure of 2,6- $\mathrm{Mes}_{2} \mathrm{C}_{6} \mathrm{H}_{3} \mathrm{SbCl}_{2}(\mathbf{1}) \cdot 0.5 \mathrm{C}_{4} \mathrm{H}_{10} \mathrm{O}$ showing $20 \%$ probability displacement ellipsoids and the atom numbering (ether molecule not shown). Crystal data for $\mathbf{1} \cdot 0.5 \mathrm{C}_{4} \mathrm{H}_{10} \mathrm{O}\left(\mathrm{C}_{52} \mathrm{H}_{60} \mathrm{Cl}_{4} \mathrm{O}_{1} \mathrm{Sb}_{2}\right): \mathrm{M}_{\mathrm{c}}=1086.36$, monoclinic space group $P 2_{1}$, $a=10.657(2), b=17.087(3), c=13.974(3) \AA, \beta=93.371(5)^{\circ}, V=2540.3(9) \AA^{3}, Z=2, \rho_{\text {calcd }}$ $=1.420 \mathrm{mg} \mathrm{m}^{-3}$, crystal dimensions $0.44 \times 0.40 \times 0.09 \mathrm{~mm}^{3}$. Of 31220 reflections collected at $173 \mathrm{~K}, 15013$ (13553) were observed and used for all calculations. After absorption correction the structure was solved by direct methods and refined anisotropically on $\mathrm{F}^{2}$. Final residuals $R_{1}=0.0329, w R_{2}=0.0822(I>2 \sigma(I)) ; R_{1}=0.0398, w R_{2}=0.0882$ (all data). GooF $=1.051$, 609 parameters. Crystallographic data (excluding structure factors) for the structure has been deposited with the Cambridge Crystallographic Data Centre as supplementary publication no. CCDC-638416. Selected bond parameters [ $\left[\AA^{\circ}{ }^{\circ}\right]$ : Sb1-C11 2.385(4), Sb1-Cl2 2.380(2), Sb1C10 2.161(3), Sb1‥Cl4 3.407(6), Sb2-Cl3 2.374(2), Sb2-Cl4 2.389(4), Sb2-C40 2.177(3), Sb2‥Cl1 3.432(6), C10-Sb1-Cl1 101.94(8), C10-Sb1-Cl2 97.07(8), Cl1-Sb1-Cl2 91.34(4), C40-Sb2-Cl3 95.13(8), C40-Sb2-Cl4 103.53(8), Cl3-Sb2-Cl4 91.01(4). 


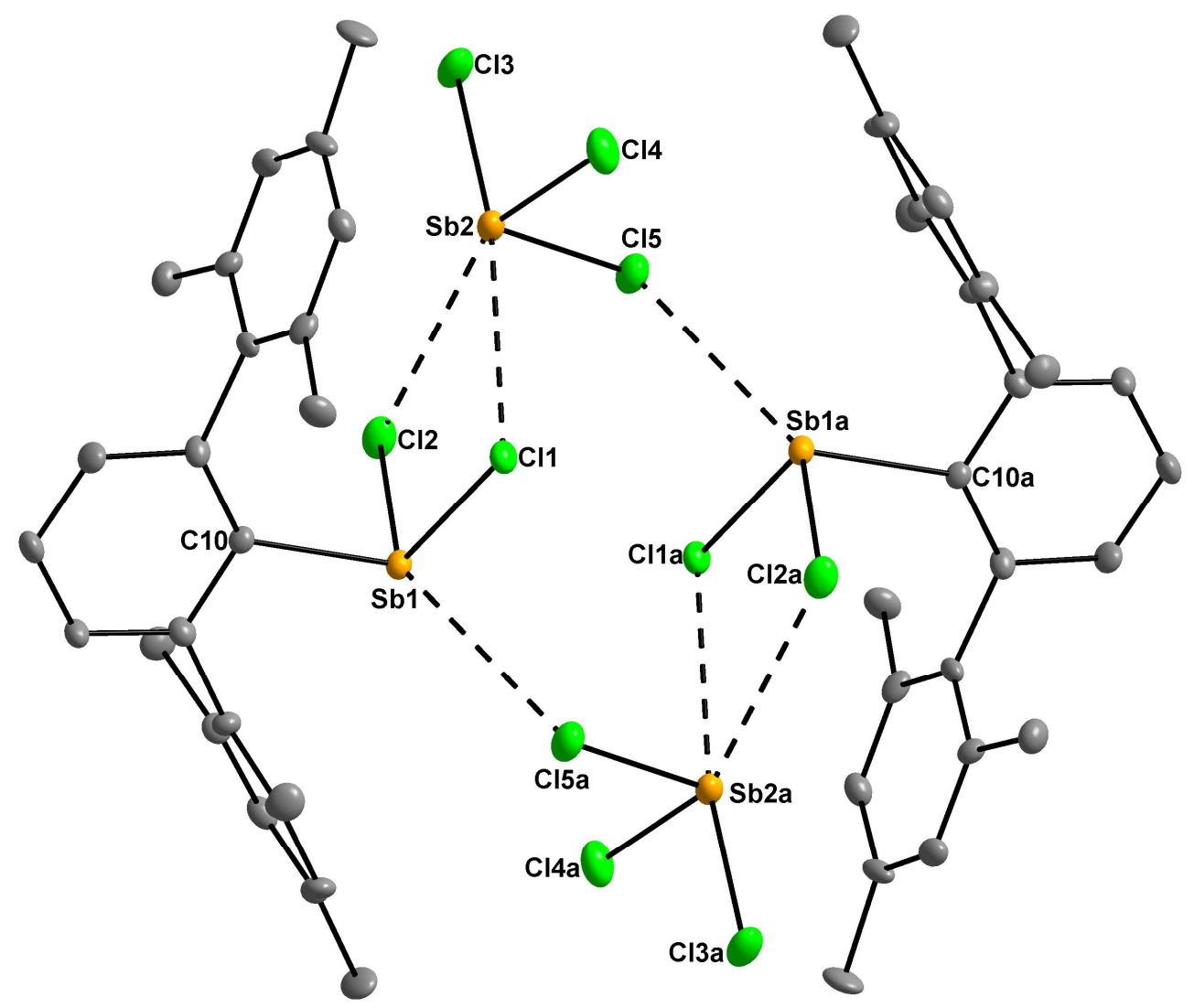

Figure S2. Molecular structure of 2,6- $\mathrm{Mes}_{2} \mathrm{C}_{6} \mathrm{H}_{3} \mathrm{SbCl}_{2}(\mathbf{1}) \cdot \mathrm{SbCl}_{3}$ showing $20 \%$ probability displacement ellipsoids and the atom numbering (independent molecules not shown). Crystal data for $1 \cdot \mathrm{SbCl}_{3}\left(\mathrm{C}_{22} \mathrm{H}_{25} \mathrm{Cl}_{5} \mathrm{Sb}_{2}\right): \mathrm{M}_{\mathrm{c}}=710.21$, monoclinic space group $P 2_{1} / \mathrm{c}, a=38.306(6), b=8.7273(14), c=24.836(4) \AA, \beta=99.759(4)^{\circ}, V=8183(2) \AA^{3}, Z=$ $18, \rho_{\text {calcd }}=1.788 \mathrm{mg} \mathrm{m}^{-3}$, crystal dimensions $0.50 \times 0.44 \times 0.24 \mathrm{~mm}^{3}$. Of 63456 reflections collected at $173 \mathrm{~K}, 14396$ (8644) were observed and used for all calculations. After absorption correction the structure was solved by direct methods and refined anisotropically on $\mathrm{F}^{2}$. Final residuals $R_{1}=0.0787, w R_{2}=0.1833(I>2 \sigma(I)) ; R_{1}=0.1371, w R_{2}=0.2142$ (all data). $\mathrm{GooF}=0.966,839$ parameters. Crystallographic data (excluding structure factors) for the structure has been deposited with the Cambridge Crystallographic Data Centre as supplementary publication no. CCDC-638417. Selected bond parameters [Å, $\left.{ }^{\circ}\right]$ : Sb1-C11 2.385(5), Sb1-Cl2 2.377(5), Sb1-C10 2.182(10), Sb2-Cl3 2.374(6), Sb2-Cl4 2.337(5),

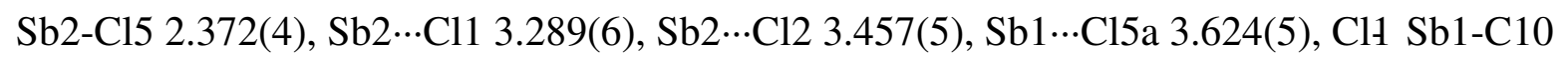
101.18(28), Cl2-Sb1-C10 94.86(29), Cl1-Sb1-Cl2 96.19(11), Cl3-Sb2-Cl4 96.57(13), Cl3Sb2-Cl5 93.01(13), Cl4-Sb2-Cl5 93.36(13). 

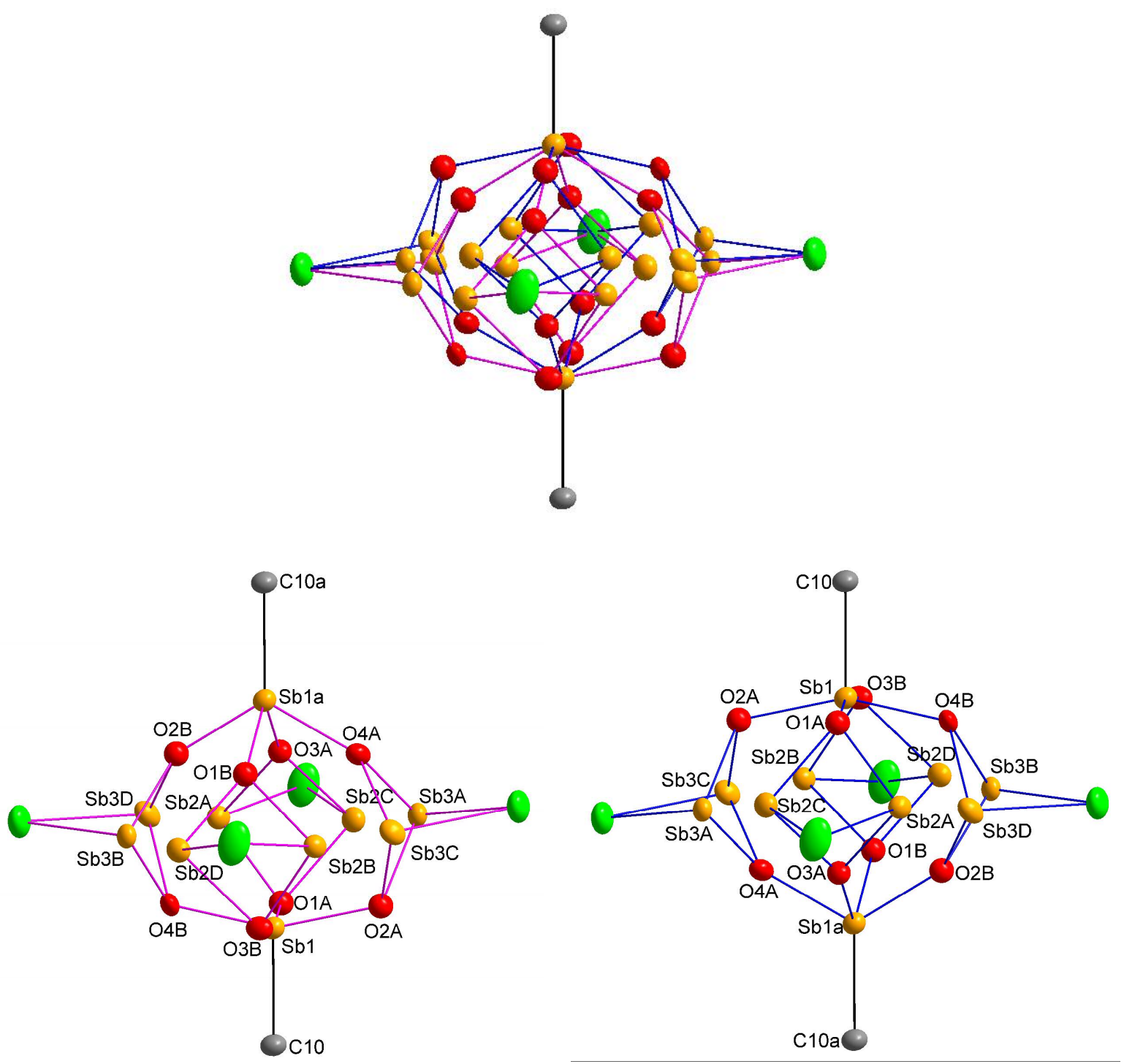

Figure S3. Inorganic core of $\left(2,6-\mathrm{Mes}_{2} \mathrm{C}_{6} \mathrm{H}_{3} \mathrm{Sb}^{\mathrm{V}}\right)_{2}\left(\mathrm{ClSb}^{\mathrm{III}}\right)_{4} \mathrm{O}_{8}(3)$ illustrating the two major disordered fragments and the numbering scheme. All oxygen atoms were refined with split occupancies of 0.5. The antimony atoms $\mathrm{Sb} 2$ and $\mathrm{Sb} 3$ are further disordered and were refined with split occupancies of 0.3 (Sb2A, Sb2B, Sb3A, Sb3B) and 0.2 (Sb2C, Sb2D, Sb3C, Sb3D). 


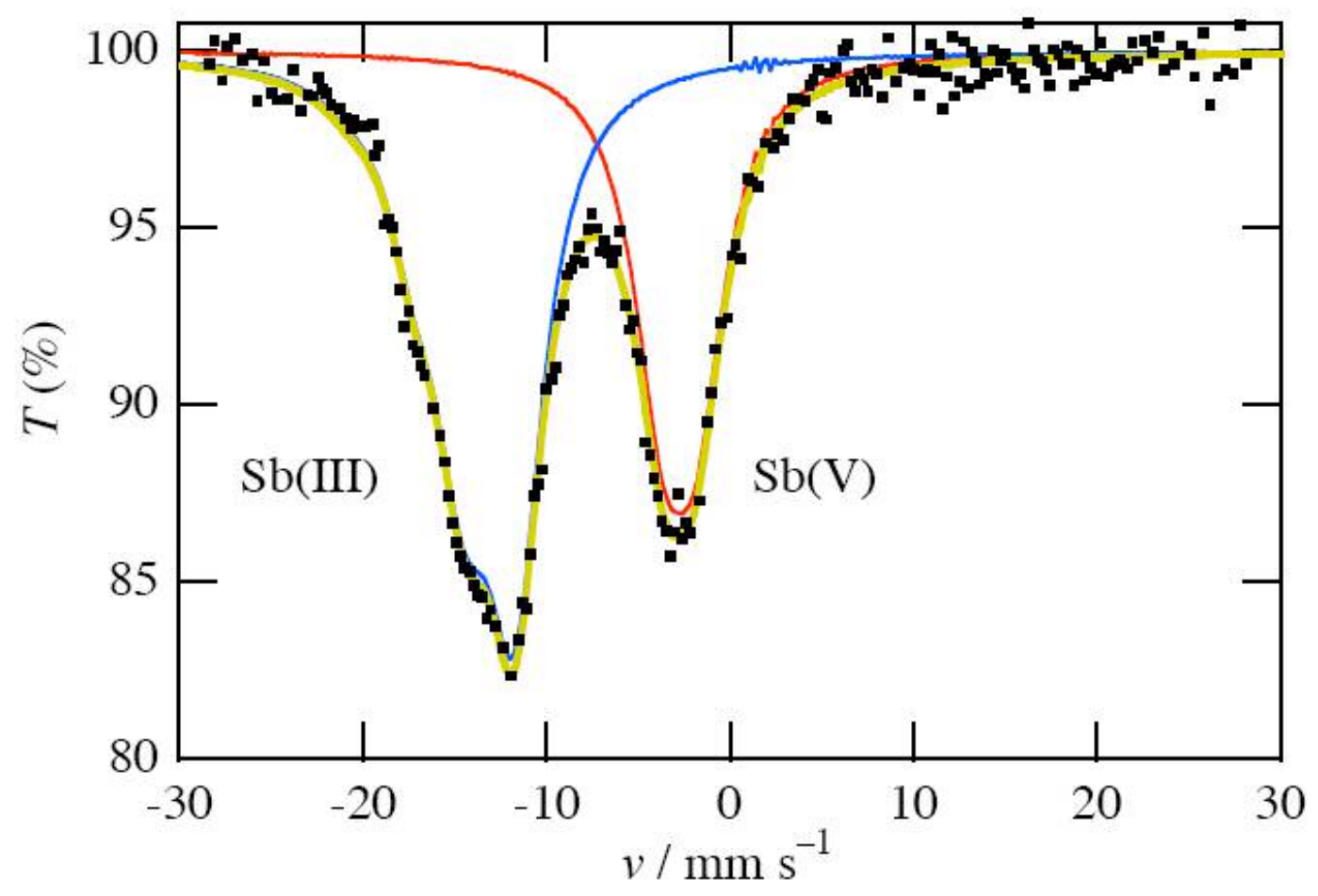

Figure S4. $\quad{ }^{121} \mathrm{Sb}$ Mößbauer spectrum of $\left(2,6-\mathrm{Mes}_{2} \mathrm{C}_{6} \mathrm{H}_{3} \mathrm{Sb}^{\mathbf{V}}\right)_{2}\left(\mathrm{ClSb}^{\text {III }}\right)_{4} \mathrm{O}_{8}(3)$ collected at $12 \mathrm{~K}$ with Wissel Mößbauer driving system 1200 using a $\mathrm{Ca}^{121 \mathrm{~m}} \mathrm{SnO}_{3}$ source $(16 \mathrm{MBq})$ and a pure Ge detector. The ${ }^{121} \mathrm{Sb}$ isomer shifts $(\delta)$ are given relative to InSb source $\left(-8.62 \mathrm{~mm} \mathrm{~s}^{-1}\right.$ relative to $\mathrm{Ca}^{121 \mathrm{~m}} \mathrm{SnO}_{3}$ source;). Data deconvolution: $\mathrm{Sb}(\mathrm{V})$ site: $\delta=5.86 \mathrm{~mm} \mathrm{~s}^{-1} ; e^{2} q Q$ (quadrupole coupling constant $)=8.9 \mathrm{~mm} \mathrm{~s}^{-1} ; \eta$ (asymmetry parameter $)=0.99$; $\Gamma_{\exp }($ experimental linewidth $)=3.30 \mathrm{~mm} \mathrm{~s}^{-1}$; area $38 \%$; Sb(III) site: $\delta=-4.86 \mathrm{~mm} \mathrm{~s}^{-1} ; e^{2} q Q=$ $19.1 \mathrm{~mm} \mathrm{~s}^{-1} ; \eta=0.19 ; \Gamma_{\exp }=3.30 \mathrm{~mm} \mathrm{~s}^{-1}$; area $62 \%$. 